\title{
On-Line Partial Discharge Localization in Power Cables Based on Electromagnetic Time Reversal Theory - Numerical Validation
}

\author{
A. Ragusa, Member, IEEE. H. Sasse and A. Duffy, Fellow, IEEE,
}

\begin{abstract}
This paper presents the numerical validation of a new on-line location method of partial discharge (PD) in cables of Medium Voltage (MV) power networks based on the electromagnetic time reversal (EMTR) theory. Because PD events are a symptom of cable insulation degradation, PDs localization is a key topic for fault prevention on power networks, increasing their reliability and resilience and to guaranteeing electricity security. A description of the proposed EMTR PD location method is given and its effectiveness and accuracy are analyzed in lines with both homogeneous and inhomogeneous power cables. The accuracy of PD location methods is strongly affected by the distortion of the PD pulses during their propagation on power lines, caused mainly by the skin effect. For this reason, the accuracy of the proposed location method is analyzed using a lossy model of power networks that is able to reproduce PD signals distortion. The model used, realized using the transmission line matrix (TLM) method, is also presented here and theoretically validated.
\end{abstract}

Index Terms-Cable system, partial discharges, fault prevention, electromagnetic time reversal, power system protection, reliability, TLM modeling.

\section{INTRODUCTION}

$\mathrm{F}$ AILURES of power cables' insulation in distribution and transmission networks cause effects ranging from faults to blackouts and supply interruption, leading to severe social and economic consequences. Because statistics indicate that more than $85 \%$ of equipment failures are linked to insulation failure [1], the adoption of on-line diagnostic methods of insulation degradation is a powerful solution to improve network resilience.

Cable insulation deterioration is often caused by partial discharge (PD) events that are localized electrical discharges, starting in discontinuities or defects of the insulation system, that only partially bridge the insulation between conductors [2]. Since PD is widely regarded as one of the best 'early warning' indicators of cable failure [3], the on-line PD location is the most suitable monitoring method to assess network integrity [4]-[10]. The adoption of on-line PD location methods is, in

This project has received funding from the European Union's Horizon 2020 Research and Innovation Programme under the Marie Skłodowska-Curie grant agreement No 838681 .

The authors are grateful for the numerous discussions with Prof. F. Rachidi from École Polytechnique Fédérale de Lausanne, Switzerland, Prof. M. fact, a desired feature in the protection schemes of modern networks to prevent faults and supply interruptions, enhancing network reliability and guaranteeing electricity security[11].

The most adopted on-line PD location methods are reflectometry or traveling wave-based techniques[5]-[10], based on the fact that a PD event produces electromagnetic waves that travel in either direction towards the cable ends. The most used methods are multi-end measurement techniques where the incident wave and the reflected waves from the cable ends, produced by PD events, are simultaneously measured at different points of the line. From the times of arrival of the measured signals, the PD source is located (time of arrival, ToA, methods). The practical implementation of multi-end ToA methods is difficult because synchronization is needed. Moreover, their accuracy is influenced by the distortion phenomenon that characterize the propagation of PD signals on power cables and by the presence of electromagnetic interference on power networks. Wavelet techniques [12][13] have been used to overcome some of the shortcomings associated with the reflectometry method, but they are characterized by a considerable amount of computational effort. EMTR (Electromagnetic Time Reversal) theory [14] has been recently used to locate sources of electromagnetic disturbances in power systems, and methods to locate lightning strikes, lightning-originated flashovers [15][16] and faults[14][17] have been developed from it. The advantages of the EMTR methods [12], with respect to traditional location techniques, are the applicability to inhomogeneous and complex networks, the robustness against the presence of noise and the ability to use a single observation point.

A new method for the on-line localization of PDs based on EMTR theory was proposed by the Authors in [18] where the basic design of the method is described and its theorical effectiveness to locate $\mathrm{PD}$, using only one observation point, has been demonstrated for lines with homogeneous cables. The basic steps of the method are shown in Fig. 1 and described here:

1. Recording of the electromagnetic signal generated by the $\mathrm{PD}$ at one observation point (OP) along the line.

2. Simulating the time-reversed injection of the measured PD

Rubenstein from the School of Management and Engineering Vaud, Yverdonles-Bains, Switzerland and with Marc Foxall and Malcolm Seltzer-Grant from HVPD Ltd. In Salford, UK.

A. Ragusa, H. Sasse, and A. Duffy are with the School of Engineering and Sustainable Development, De Montfort University, Leicester LE1 9BH, U.K. (e-mail: antonella.ragusa@dmu.ac.uk; hgs@dmu.ac.uk; apd@dmu.ac.uk). 
signal for different guessed PD locations (GPDLs) using a lossless 1D Transmission Line Matrix (TLM) model.

3. Locating the PD source by identifying the GPDL characterized by the highest energy concentration.

In practical implementation, the first step of the procedure, is carried out experimentally. In [18], to design the method, a first validation of the procedure was made using a lossless model to perform the simulation in the direct time (DT) domain of step 1. Hence, in [16], the behavior of a real network has not been considered and the DT simulation does not reproduce the variation with frequency of the line impedance and propagation constant, which causes the PD pulses' distortion [19]-[21] affecting effectiveness and accuracy of the location methods.

In this paper, a more exhaustive numerical validation of the method is given using a lossy TLM model of the line for the DT simulation, that is able to reproduce the PD signal's distortion. The developed lossy TLM model is described and theoretically validated. The designed EMTR PD location method is presented, and its effectiveness and accuracy are analyzed.

The paper is organized as follows. In Section 2 the EMTR theory for locating PDs is introduced. Section 3 describes the developed lossy 1D TLM model and in Section 4 the TR simulation and the PD localization procedure are presented. Finally, in Section 5 the effectiveness and accuracy of the method is investigated in networks with both homogeneous and inhomogeneous cables.

\section{EMTR THEORY TO LOCATE PARTIAL Discharges}

The PD location method designed using EMTR theory [18] is based on the invariance under time reversal of the Telegrapher's Equations for non-dissipative lines[14]. For non-dissipative lines, the telegrapher's equations are given by:

$$
\begin{aligned}
& \frac{\partial v(x, t)}{\partial x}+L \frac{\partial i(x, t)}{\partial t}=0 \\
& \frac{\partial i(x, t)}{\partial x}+C \frac{\partial v(x, t)}{\partial t}=0
\end{aligned}
$$

where $L$ and $C$ are the per unit length series inductance and shunt capacitance of the line. The propagation speed, $u$, and a characteristic impedance, $Z_{0}$, of the line are given by:

$$
u=\frac{1}{\sqrt{L C}} \quad ; \quad Z_{0}=\sqrt{L / C}
$$

Applying the time-reversal transformation, equations (1) become:

$$
\begin{aligned}
& \frac{\partial v(x,-t)}{\partial x}+L \frac{\partial(-i(x,-t))}{\partial(-t)}=0 \\
& \frac{\partial(-i(x,-t))}{\partial x}+C \frac{\partial(v(x,-t))}{\partial(-t)}=0
\end{aligned}
$$

These are identical to equations (1), except for that the current that has changed sign (see [14] for a discussion on the necessity of the sign change). The invariance under time reversibility

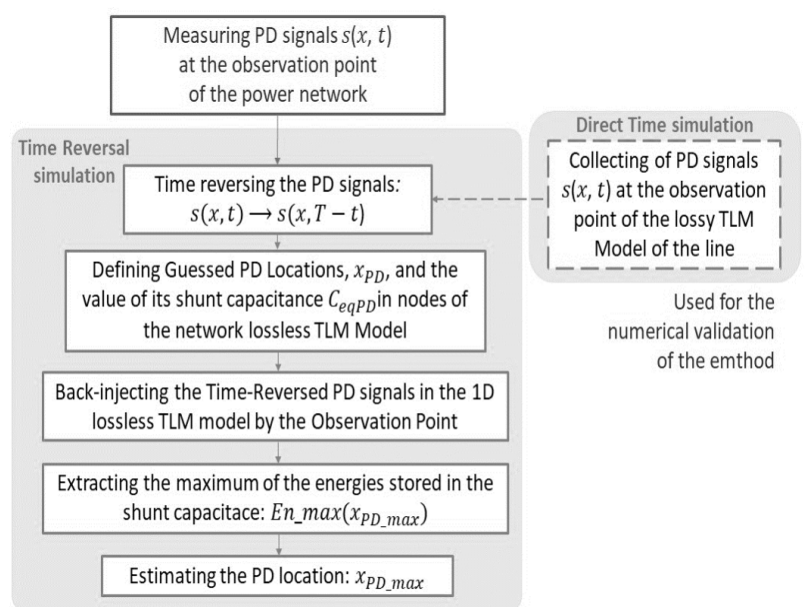

Fig. 1. EMTR-based method for the on-line localization of PDs [18].

implies that the voltage and current, $v(x, t)$, and $i(x, t)$, and their symmetric values in time, $v(x,-t)$, and $i(x,-t)$, are both solutions of the telegrapher's equations. The time reversibility of the propagation equations and the spatial correlation property of the EMTR theory allow the refocusing of the time reversed backpropagated PD signals into its original location.

The designed EMTR PD location method is described in Fig.1 [18]. To simulate the time reversal (TR) propagation of the PD signals, the TLM method has been chosen for its flexibility, high efficiency, and its numerical stability. In particular, a 1D lossless TLM model has been developed to perform the TR simulations to locate PD sources. The use of the EMTR method to localize PDs in real power lines is based on the measurement of the PD signal $s(x, t)$, at an observation point (OP) of the line. Then, the PD signal is time reversed and back injected in the lossless 1D TLM model of the line and the TR simulations are performed to localize the source. Here, with the aim of numerically validating the method, a model of the line is also used to reproduce the system behavior in the direct time (DT) domain. During the DT simulation, a PD source is simulated at a point $x_{P D}$ of the line and the generated PD signal propagates from the source towards the line terminations where the signal is collected. In [18] the authors used a lossless 1D TLM model also to perform the DT simulation. But the nonzero resistance of a real cable affects the signal propagation distorting the PD signal. In real networks, in fact, if $R>0$, both $R$ and $L$ vary with the frequency, and the same occurs for $C$ and $G$ if $G>0$ [20]. In order to model the PD distortion in an approximate but simple way, the effect of $\mathrm{G}$ and of the losses in the semiconductor layers are ignored [20] [21].

Considering a two-wire line (or a coaxial cable), with conductors $\mathrm{a}$ and $\mathrm{b}$, the line inductance is given by the sum of three components: $L^{\prime}$ that is the inductance of the line if the conductors had an infinite conductivity, hence with zero magnetic flux inside them, and $L_{a}$ and $L_{b}$ that are incremental components due to the conductors' finite conductivity. The skin effect, a nonuniformity of the current density due to the timevarying magnetic flux inside the conductors, produces an increase of the resistance and a reduction of $L_{a}$ and $L_{b}$ with frequency, while $L^{\prime}$ stays constant [20]. This phenomenon affects the behavior of the line attenuation and phase constants, becoming the main cause of signal distortion, which affects the 
effectiveness and accuracy of PD location methods. Here, a lossy model of the line is used to perform the DT simulation.

\section{PD Signal Propagation In LOSSY POWER NetWorks}

The developed model of lossy lines is described. In section 3.1 the skin effect and the PD signals' distortion are theoretically introduced. Section 3.2 describes the 1D TLM model of the PD signals' propagation in lines with skin effect.

\section{A. Skin effect in power networks}

The propagation of PD signals in lossy power lines is described by the equations:

$$
\begin{aligned}
& \frac{\partial v(x, t)}{\partial x}+L \frac{\partial i(x, t)}{\partial t}+R i(x, t)=0 \\
& \frac{\partial i(x, t)}{\partial x}+C \frac{\partial v(x, t)}{\partial t}+G v(x, t)=0
\end{aligned}
$$

where $R$ and $G$ are respectively the series resistance, $R$, and a shunt conductance, $G$, per unit length.

Under sinusoidal excitation, the solutions of equations (4) are:

$v(x, t)=V_{0}^{+} e^{-\alpha x} \cos (\omega t-\beta x)+V_{0}^{-} e^{\alpha x} \cos (\omega t+\beta x)(5.1)$

$i(x, t)=I_{0}^{+} e^{-\alpha x} \cos (\omega t-\beta x)+I_{0}^{-} e^{\alpha x} \cos (\omega t+\beta x)$

and the propagation of the sinusoidal waves are defined by the frequency dependent complex characteristic impedance, $Z_{0}$ and the propagation function, $\gamma$, of the line given by:

$$
\begin{aligned}
& Z_{0}=\sqrt{(R+j \omega L) /(G+j \omega C)} \\
& \gamma=\alpha+j \beta=\sqrt{(R+j \omega L)(G+j \omega C)}
\end{aligned}
$$

where, in equations (5)-(7), $V_{0}^{+}, V_{0}^{-}, I_{0}^{+}$and $I_{0}^{-}$are the wave amplitudes, and the signs + and - indicates waves travelling along $+x$ and $-x$ direction, $(R+j \omega L)$ and $(G+j \omega C)$ are, respectively, the distributed complex impedance and admittance of the line, $\omega=2 \pi f$ is the angular frequency and $f$ the frequency, and $\alpha$ and $\beta$ the attenuation function, in $\mathrm{dB} / \mathrm{m}$, and the phase function, in $\mathrm{rad} / \mathrm{m}$. The attenuation function, $\alpha$, defines the amplitude reduction per unit length of the sinusoidal travelling wave and it is the main cause of the PD pulse peak reduction and width increase, as shown below. While the phase function, $\beta$, is related to the phase velocity of the sinusoidal wave, $u$, as follows:

$$
u=\frac{\omega}{\beta}=\frac{2 \pi}{\beta} f
$$

For a lossy line, $\beta$ is not directly proportional to the frequency, hence $u$ is a nonlinear function of frequency. The phase velocity variability with frequency is the cause of the PD pulse dispersion, as shown next.

Consider a coaxial cable line (or other two conductor transmission line), shown in Figure 2, formed by a uniform, solid circular inner conductor, of radius $r_{a}$, and a uniform annular outer conductor, with inner radius $r_{b}$ and outer radius $r_{c}$. The two conductors have respectively a conductivity $\sigma_{a}$ and $\sigma_{b}$, and an absolute permeability $\mu_{a}$ and $\mu_{b}$. The dielectric between the conductors has an absolute permeability $\mu_{i}$.

At high frequency, an approximation of the cable distributed impedance, $\left(R_{h f}+j \omega L_{h f}\right)$, can be derived by the second-order asymptotic approximation of the modified Bessel functions that describe the current density of the conductors [20], as follows:

$$
\begin{aligned}
& \left(R_{h f}+j \omega L_{h f}\right)=j \omega L^{\prime}+\left(R_{a}+j \omega L_{a}\right)+\left(R_{b}+j \omega L_{b}\right) \approx \\
& \approx \frac{j \omega \mu}{2 \pi} \ln \frac{r_{a}}{r_{b}}+\sqrt{\omega}(1+j)\left(\frac{1}{2 \pi r_{a}} \sqrt{\frac{\mu_{a}}{2 \sigma_{a}}}+\frac{1}{2 \pi r_{b}} \sqrt{\frac{\mu_{b}}{2 \sigma_{b}}}\right)+ \\
& \quad+\frac{1}{4 \pi}\left(\frac{1}{r_{a}^{2} \sigma_{a}}-\frac{1}{r_{b}^{2} \sigma_{b}}\right)
\end{aligned}
$$

where $\sqrt{\omega \mu_{a} \sigma_{a}} \cdot r_{a} \gg 1$ and $\sqrt{\omega \mu_{b} \sigma_{b}} \cdot r_{b} \gg 1$. The first term in (9) is the impedance component due to the time-varying flux between the inner and outer conductor, with the related frequency independent inductance, $L^{\prime}$, given by:

$$
L^{\prime}=\frac{\mu_{i}}{2 \pi} \ln \frac{r_{a}}{r_{b}}
$$

The terms $\left(R_{a}+j \omega L_{a}\right)$ and $\left(R_{b}+j \omega L_{b}\right)$ are the impedance components due to a nonuniform current density caused by the time-varying magnetic flux inside the conductors, $a$ and $b . R_{a}$, $R_{b}, L_{a}$ and $L_{b}$ are the related resistances and inductances.

Rearranging (9), the high frequency impedance component of the inner conductor (the same can be done for the outer one) is given by:

$$
R_{a}+j \omega L_{a} \approx \frac{\sqrt{\omega \mu_{a} \sigma_{a}}}{2 \sqrt{2} \pi r_{a} \sigma_{a}}+\frac{1}{4 \pi r_{a}^{2} \sigma_{a}}+j \frac{\sqrt{\omega \mu_{a} \sigma_{a}}}{2 \sqrt{2} \pi r_{a} \sigma_{a}}
$$

with:

$$
\begin{aligned}
& R_{a}=\frac{\sqrt{\omega \mu_{a} \sigma_{a}}}{2 \sqrt{2} \pi r_{a} \sigma_{a}}+\frac{1}{4 \pi r_{a}^{2} \sigma_{a}}=\frac{\sqrt{\pi f \mu_{a} \rho_{a}}}{2 \pi r_{a}}+\frac{1}{4 \pi r_{a}^{2} \sigma_{a}} \\
& L_{a}=\frac{\sqrt{\omega \mu_{a} \sigma_{a}}}{2 \omega \sqrt{2} \pi r_{a} \sigma_{a}}=\frac{1}{2 \pi r_{a}} \sqrt{\frac{\mu_{a} \rho_{a}}{4 \pi f}}
\end{aligned}
$$

where $\rho_{a}$ is the resistivity of the conductor. Equations (11) show the skin effect of the conductors producing an increase of the resistance proportional to $\sqrt{f}$ and a reduction of the inductance proportional to $\sqrt{1 / f}$. Now, considering $G=0$, then a distributed complex admittance equal to $j \omega C$, from (7) and (9) and simplifying [20], the propagation constant at high frequency can be evaluated:

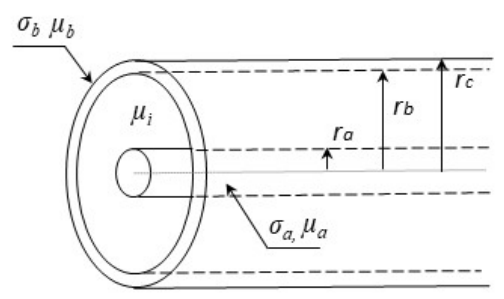

Fig. 2. Geometric characteristics of a coaxial cable. 


$$
\gamma \approx j \omega \sqrt{L^{\prime} C}+\frac{\xi}{2 \sqrt{2}} \sqrt{\frac{C}{L^{\prime}}}(1+j) \sqrt{\omega}
$$

where:

$$
\xi=\left(\frac{1}{2 \pi r_{a}} \sqrt{\frac{\mu_{a}}{\sigma_{a}}}+\frac{1}{2 \pi r_{b}} \sqrt{\frac{\mu_{b}}{\sigma_{b}}}\right)
$$

From (12), the frequency dependence of the attenuation, phase function and phase velocity can be evaluated:

$$
\begin{aligned}
& \alpha \approx \frac{\xi}{2 \sqrt{2}} \sqrt{\frac{C}{L^{\prime}}} \sqrt{\omega} \\
& \beta \approx \omega \sqrt{L^{\prime} C}+\frac{\xi}{2 \sqrt{2}} \sqrt{\frac{C}{L^{\prime}}} \sqrt{\omega} \\
& u \approx \frac{1}{\sqrt{L^{\prime} C}+\frac{\xi}{2 \sqrt{2 \omega}} \sqrt{\frac{C}{L^{\prime}}}}
\end{aligned}
$$

Equations (13) and (15) show that the higher frequency waves are attenuated more quickly than the lower frequency waves and that the higher frequency waves propagate more quickly than lower ones. These two phenomena affect the PD signals propagation: the separation and the different attenuations of the frequency components reduces the pulse frequency bandwidth causing distortion.

Using the asymptotic approximation of the high frequency distributed impedance $\left(R_{h f}+j \omega L_{h f}\right)$ of the line given in (9.1), valid for frequencies higher than the skin effect demarcation frequency, defined as the frequency from where the skin effect can't be neglected, it is possible to describe the distributed complex impedance of a coaxial cable, $(R+j \omega L)$, as the sum of a low frequency impedance, $\left(R_{l f}+j \omega L_{l f}\right)$ and a high frequency one:

$$
(R+j \omega L) \approx\left(R_{l f}+j \omega L_{l f}\right)+\left(R_{h f}+j \omega L_{h f}\right)
$$

where [22]:

$$
\begin{aligned}
R_{l f} & =R_{d c a}+R_{d c b}=\frac{1}{\pi r_{a}^{2} \sigma_{a}}+\frac{1}{\pi\left(r_{c}^{2}-r_{b}^{2}\right) \sigma_{b}} \\
L_{l f} & =L^{\prime}+L_{l f a}+L_{l f b}= \\
& =L^{\prime}+\frac{\mu_{a}}{8 \pi}+\frac{\mu_{b}}{2 \pi}\left[\frac{r_{c}^{4} \ln \left(r_{c} / r_{b}\right)}{\left(r_{c}^{2}-r_{b}^{2}\right)}+\frac{r_{b}^{2}-3 r_{c}^{2}}{4\left(r_{c}^{2}-r_{b}^{2}\right)}\right]
\end{aligned}
$$

where $R_{d c a}$ and $R_{d c b}$ are the DC resistance of the inner and outer conductors and $L_{l f a}$ and $L_{l f b}$ the inductance at low frequency, where it is possible to assume a radially uniform current density distribution inside the conductors so to neglect the skin effect. The demarcation frequency is given by the intersection of the $R_{h f}$ high frequency asymptote and the value of the $R_{l f}$. In the following section, a TLM model, able to describe the skin effect of a transmission line, is developed and described.

\section{B. ID TLM model of PD signal propagation in lossy lines}

The TLM method [23], chosen for its flexibility, high efficiency, and its numerical stability, is used to model lines with skin effect and to describe the PD signal propagation.

As explained previously, the skin effect causes the PD signal distortion reducing its frequency bandwidth, so the transmission line behaves as a low pass filter.

To represent the low pass characteristic of the line, a compact ladder model has been proposed in [24][25]. The skin effect produced by the nonuniform distribution of the current density inside the conductor at the higher frequencies, is represented by dividing the conductor cross section into 4 concentric rings and modelling each of them with a ladder network of resistances, $R_{i}$ and inductances, $L_{j}$, with $i=1,2,3,4$ and $j=1,2,3$. The ladder resistances and inductances are evaluated starting from the knowledge of the dc resistance of the conductor, $R_{d c}$, and of its internal inductance at the low frequency, $L_{l}$, (lower than the demarcation frequency) and using a constant ratio, $R R$ and $L L$ among the resistances and the inductances, respectively. Then the ladder parameters are given by:

and:

$$
R_{i}=R R \cdot R_{i+1} ; L_{j}=L L \cdot L_{j+1}
$$

$$
R_{1}=\alpha_{R} R_{d c} \quad \text { and } \quad L_{1}=\alpha_{L} L_{l f}
$$

The value of $\alpha_{R}$ and $\alpha_{L}$ is defined in relation with the maximum frequency of interest, $f_{m}$, [24]:

$$
\alpha_{R}=0.53 \frac{r}{\delta_{m}}=0.53 r \sqrt{\pi f_{m} \mu_{0} \sigma} ; \alpha_{L}=0.315 \alpha_{R}
$$

where $\delta_{m}$ is the skin depth and $\sigma$ and $\mu_{0}$ are the conductivity and the absolute permeability of the conductor. The $R R$ and $L L$ values can be evaluated using the knowledge of $\alpha_{R}$ and $\alpha_{L}$ and considering that the ladder dc resistance and low frequency inductance must be equal to $\mathrm{R}_{l}$, and $L_{l}$, respectively [24].

In a coaxial cable, the skin effect is modelled in both the inner and outer conductor, defining a ladder circuit for each of them.

Figure 3 shows the skin effect model for a section of length $\Delta x$ of the cable. The resistances, $R_{i a}, R_{i b}$, and inductances, $L_{j a}, L_{j b}$, of the inner, $a$, and outer, $b$, conductors are evaluated by using (17)-(19), with $R_{d c a}, R_{d c b}$, and $L_{l f a}, L_{l f b}$, given by (16.2) - (16.3).

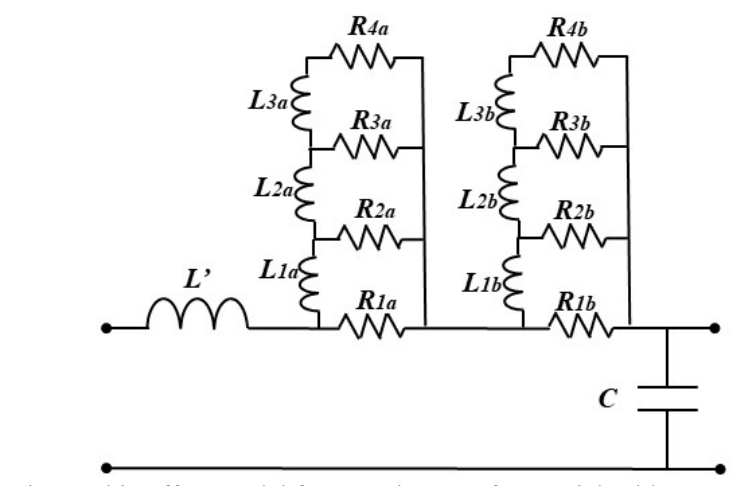

Fig. 3. Skin effect model for a section $\Delta x$ of a coaxial cable. 
The circuit in Figure 3 models the total complex distributed impedance of the line defined by equations (16).

The TLM method is used to model the power line with skin effect. The TLM method is a differential equation-based method, operating in the time-domain, that discretizes a power line, of length 1 , into a series of $\mathrm{N}$ segments or nodes, of length $\Delta x$, as shown in Figure 4(a). Each $L C$ section of the line can be represented by a transmission line with a propagation speed, $u$, and a characteristic impedance, $Z_{0}$, given by relations (2), and a transit time $\Delta t$ given by:

$$
\Delta t=\frac{\Delta x}{u}=\Delta x \cdot \sqrt{L C}
$$

Connecting the $N$ sections, the TLM equivalent model of the line shown in Figure 4(b) is obtained. At each node, the wave pulses, $V_{n}(k)$, are scattered and propagate in the line, generating incident voltages, $V L_{n}^{i}(k), V R_{n}^{i}(k)$, and reflected voltages, $V L_{n}^{r}(k), V R_{n}^{r}(k)$, respectively on the left and on the right side of the node $n$. Replacing the lines to the right and to the left of the node by their Thevenin equivalent circuits, shown in Figure 4.(c), the voltage $V_{n}(k)$, and the current, $I_{n}(k)$, at time $k$, are evaluated in each node $n$. To model the skin effect, each node of figure 4(a) is represented by the circuit model of Figure 3. The $L^{\prime} C$ section is represented by a transmission line while the ladder inductors are modeled using the TLM stub model of an inductor [23]. Figure 5 shows the Thevenin equivalent circuit of the line with skin effect. In Figure 5, each stub model of the inductors, is characterized by an impedance, $Z_{j a, b}$, of, respectively, the inner conductor, $a$, and the outer conductor, $b$, equal to:

$$
Z_{j a, b}=\frac{2 L_{j a, b}}{\Delta x} \quad \text { with } \quad j=1,2,3
$$

and the voltage $V_{j a}^{i}, V_{j b}^{i}, I_{j a}^{i}, I_{j b}^{i}$, with $j=1,2,3$, are the incident voltage at the stabs and the current at the ladder circuits.

The equations at the ladder circuits are given by:

$$
\left[\begin{array}{c}
2 V L_{n}^{i}(k)-2 V R_{n}^{i}(k) \\
2 V_{1 a}^{i}(k) \\
2 V_{2 a}^{i}(k) \\
2 V_{3 a}^{i}(k) \\
2 V_{1 b}^{i}(k) \\
2 V_{2 b}^{i}(k) \\
2 V_{3 b}^{i}(k)
\end{array}\right]-\left[Z_{s e}\right] \cdot\left[\begin{array}{c}
I_{n}(k) \\
I_{1 a}(k) \\
I_{2 a}(k) \\
I_{3 a}(k) \\
I_{1 b}(k) \\
I_{2 b}(k) \\
I_{3 b}(k)
\end{array}\right]=0
$$

where $Z_{s e}$ is the skin effect matrix given by (23).

From (22), inverting the matrix $Z_{s e}$, the currents can be evaluated. The, the voltages at the node $n$ at the time step $k$, can

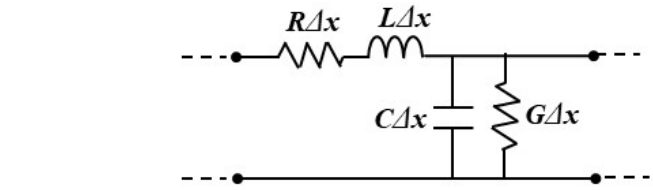

(a)

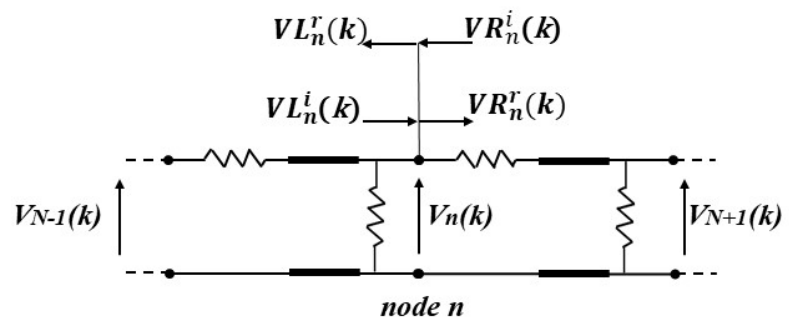

(b)

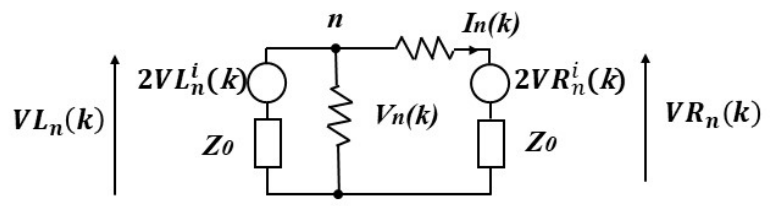

(c)

Fig. 4 Equivalent circuit of a power line node (a), its TLM model (b), and the Thevenin equivalent circuit (c) at the node $n$.

be evaluated as follows:

$$
\begin{aligned}
& V_{n}(k)=2 V R_{n}^{i}(k)+I_{n}(k)\left(Z_{0}+R_{1 a}\right)-I_{1 a} R_{1 a} \\
& V L_{n}(k)=V_{n}(k) \\
& V R_{n}(k)=2 V R_{n}^{i}(k)+I_{n}(k) Z_{0} \\
& V_{j a}=2 V_{j a}^{i}-I_{j a} Z_{j a} \quad \text { with } j=1,2,3 \\
& V_{j b}=2 V_{j b}^{i}-I_{j b} Z_{j b} \quad \text { with } j=1,2,3
\end{aligned}
$$

The voltages at the node $n$ are defined by the incident voltages at the time step $k$, as (24) show. The incident voltages at the time $k+1$ are evaluated using the node condition at time $k$. The incident voltages at the node $n$ are, in fact, coincident with the reflected voltages from the nodes $n-1$ and $n+1$ at time step $k$, as follows:

$$
\begin{aligned}
& V L_{n}^{i}(k+1)=V R_{n-1}^{r}(k) \\
& V R_{n}^{i}(k+1)=V L_{n+1}^{r}(k)
\end{aligned}
$$

While the incident voltage at the stub model of the inductor are given by equations (26) [23]:

$$
\begin{aligned}
& V_{j a}^{i}(k+1)=-V_{j a}^{r}(k) \\
& V_{j b}^{i}(k+1)=V_{j b}^{r}(k)
\end{aligned}
$$

$$
Z_{s e}=\left[\begin{array}{ccccccc}
2 Z_{0}+R_{1 a} & -R_{1 a} & 0 & 0 & -R_{1 b} & 0 & 0 \\
-R_{1 a} & R_{1 a}+R_{2 a}+Z_{1 a} & -R_{2 a} & 0 & 0 & 0 & 0 \\
0 & -R_{2 a} & R_{2 a}+R_{3 a}+Z_{2 a} & -R_{3 a} & 0 & 0 & 0 \\
0 & 0 & -R_{3 a} & R_{3 a}+R_{4 a}+Z_{3 a} & 0 & 0 & 0 \\
-R_{1 b} & 0 & 0 & 0 & R_{1 b}+R_{2 b}+Z_{1 b} & -R_{2 b} & 0 \\
0 & 0 & 0 & 0 & -R_{2 a} & R_{2 b}+R_{3 b}+Z_{2 b} & 0 \\
0 & 0 & 0 & 0 & 0 & -R_{3 b} & R_{3 b}+R_{4 b}+Z_{3 b}
\end{array}\right]
$$




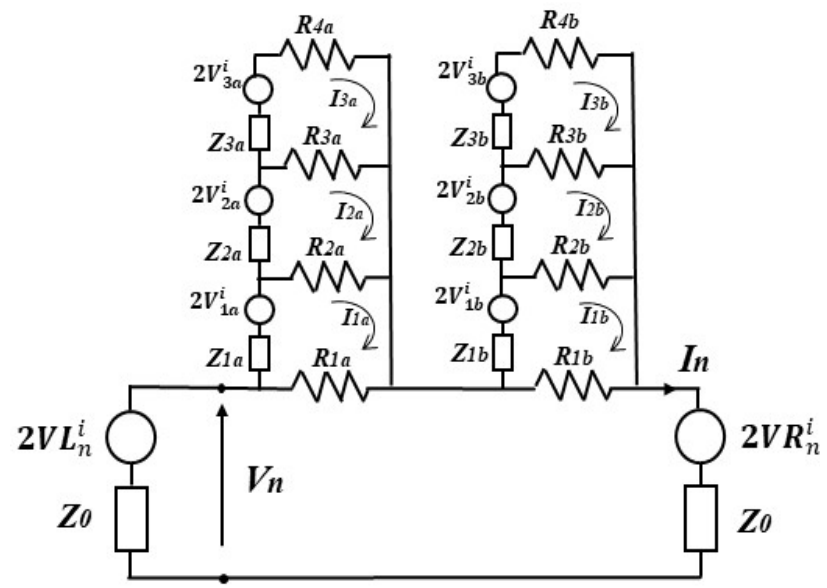

Fig. 5. Thevenin equivalent circuit of the TLM model of a node $n$ of a line with skin effect.

The node voltages are scattered in the line as follows:

$$
\begin{aligned}
& V L_{n}^{r}(k)=V L_{n}(k)-V L_{n}^{i}(k) \\
& V R_{n}^{r}(k)=V R_{n}(k)-V R_{n}^{i}(k) \\
& V_{j a}^{r}(k)=V_{j a}(k)-V_{j a}^{i}(k) \quad \text { with } j=1,2,3 \\
& V_{j b}^{r}(k)=V_{j b}(k)-V_{j b}^{i}(k) \quad \text { with } j=1,2,3
\end{aligned}
$$

where $V_{j a, b}^{r}$ are the reflected voltage at the stub inductors.

Two TLM models, for homogeneous and inhomogeneous lines, have been developed to perform the simulation of the PD signal propagation in the direct time domain to give a more accurate validation of the designed EMTR PD location method.

For the inhomogeneous case, the line is formed from two cable sections, respectively $l_{1}$ and $l_{2}$ long, with characteristic impedance $Z_{01}$ and $Z_{02}$, and the propagation speeds, $u_{1}$ and $u_{2}$. To maintain the same time-step $\Delta t$ throughout the simulation, the length, $\Delta x_{1}, \Delta x_{2}$, of the two cable sections are given by:

$$
\begin{array}{ll}
\Delta x_{1}=u_{1} \Delta t & \text { for the cable section of length } l_{l} \\
\Delta x_{2}=u_{2} \Delta t & \text { for the cable section of length } l_{2}
\end{array}
$$

The voltages and currents at the nodes are evaluated by using the relations (22)-(27) in both the line sections, considering, in the discontinuity node, the variation of the impedance of the line from the right side and from the left side of the node.

\section{PD Source Localization AND Time REVERSAL PROPAGATION}

The basic steps of the EMTR PD location method are shown in Figure 1 and briefly described here.

A schematic representation of the line under study is shown in Figure 6. The line is connected to the load impedances, $Z_{I}$ and $Z_{2}$. Under the hypothesis that they are power transformers, the impedance $Z_{1}$ and $Z_{2}$ are equal to $100 \mathrm{k} \Omega$, which is a reasonable approximation for the power transformers' input characteristic impedance at high frequencies. The PD event occurs in a point $x_{P D}$ along the line, inside the insulation between the inner and outer conductor (ground connected), and generates an impulsive signal, represented, for the purpose of illustration, by a double exponential signal.

During the DT simulation, performed with the described lossy TLM model, the PD signal, $s(x, t)$, is recorded at the observation point (OP) shown in Figure 6, for an observation period $T$, and it is time reversed as follows:

$$
s_{i}(x, t) \rightarrow s_{i}\left(x, t^{\prime}\right) \text { with } t^{\prime}=T-t
$$

Then, the time reversed signal is back injected, from the same OP, into the 1D lossless TLM model of the same line for the time reversal (TR) simulations [16]. Several TR simulations are performed, considering, in each simulation, a guessed $\mathrm{PD}$ location (GPDL) in a point of the line. The GPDL position is changed, in each TR simulation, to explore the whole line.

A schematic representation of the line used for the TR simulations is shown in Figure 7.

The same scheme is used for the inhomogeneous one.

The TLM model of the GPDL node reproduces the line transversal capacitance between the inner and outer conductor modified by the PD event. modified by the PD event. The three capacitors model of PD [26] and the generalized PD model of Niemeyer [27] have been used to define the GPDL impedance [18]. This impedance has been modelled using a stub capacitor at the node. For each TR simulation the energy, $E_{n}$, stored in the transversal impedance is evaluated, normalized with respect to the maximum energy:

$$
E n=\frac{\frac{1}{2} C \sum_{k=1}^{M} V_{G P D L}^{2}(k)}{\frac{1}{2} C \sum_{k=1}^{M} V_{G P D L}^{2} m} \text { with } \quad M=T / \Delta t
$$

where $V_{G P D L \_}(k)$ is the maximum voltage at the GPDLs and $M$ the number of the samples. The PD location will be coincident with the GPDL characterized by the maximum energy because the time reversed PD pulses will add up in phase at the real PD location during their backward propagation.

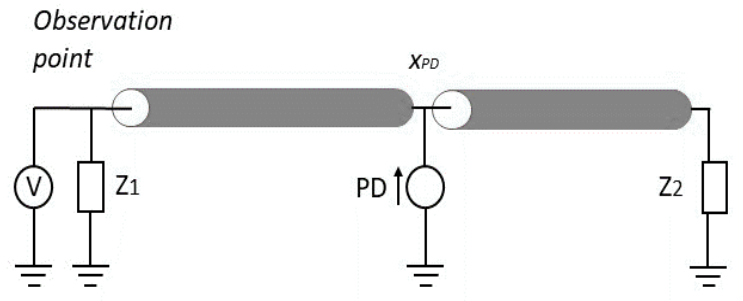

Fig. 6. Schematic representation of the system under study.

Guessed PD Locations

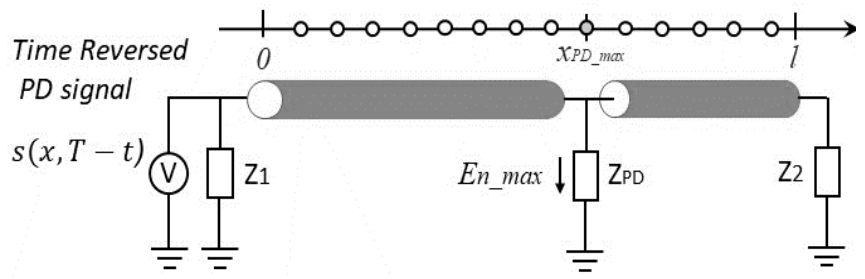

Fig. 7. Schematic representation of the line used for the TR simulations. 
TABLE 1.

CABLES CHARACTERISTICS

\begin{tabular}{|c|c|c|}
\hline Parameter & Cable 1 & Cable 2 \\
\hline $\begin{array}{l}\text { Inner conductor conductivity, } \sigma_{a} \\
\text { Outer conductor conductivity, } \sigma_{b} \\
\text { Radius of inner conductor, } r_{a} \\
\text { Inner radius of outer conductor, } r_{b} \\
\text { Outer radius of outer conductor, } r_{c} \\
\text { Relative permittivity of the insulator }\end{array}$ & $\begin{array}{cc}0.58 \cdot 10^{8} & \mathrm{~S} / \mathrm{m} \\
0.58 \cdot 10^{8} & \mathrm{~S} / \mathrm{m} \\
8.2 \cdot 10^{-3} \mathrm{~m} \\
14 \cdot 10^{-3} \mathrm{~m} \\
14.8 \cdot 10^{-3} \mathrm{~m} \\
2.2\end{array}$ & $\begin{array}{c}0.58 \cdot 10^{8} \mathrm{~S} / \mathrm{m} \\
0.58 \cdot 10^{8} \mathrm{~S} / \mathrm{m} \\
8.4 \cdot 10^{-3} \mathrm{~m} \\
14.2 \cdot 10^{-3} \mathrm{~m} \\
15 \cdot 10^{-3} \mathrm{~m} \\
2.8\end{array}$ \\
\hline
\end{tabular}

TABLE 2 .

ELECTRICAL PARAMETERS OF THE TLM MODEL.

\begin{tabular}{l|c|c}
\hline \multicolumn{1}{c}{ Parameter } & Cable 1 & Cable 2 \\
\hline Shunt capacitance, $C$ & $2.2880 \cdot 10^{-10} \mathrm{~F} / \mathrm{m}$ & $2.9670 \cdot 10^{-10} \mathrm{~F} / \mathrm{m}$ \\
Series inductance, $L$ & $1.0698 \cdot 10^{-7} \mathrm{H} / \mathrm{m}$ & $1.0500 \cdot 10^{-7} \mathrm{H} / \mathrm{m}$ \\
Characteristic impedance, $Z_{0}$ & $21.6237 \Omega$ & $19.2413 \Omega$ \\
Propagation speed, $u$ & $2.0212 \cdot 10^{8} \mathrm{~m} / \mathrm{s}$ & $1.7916 \cdot 10^{8} \mathrm{~m} / \mathrm{s}$ \\
Ladder resistances, $R_{l a}, R_{1 b}$ & $0.0379,0.1997 \Omega$ & $0.0370,0.1996 \Omega$ \\
Ladder resistances, $R_{2 a}, R_{2 b}$ & $0.0051,0.0220 \Omega$ & $0.005,0.0219 \Omega$ \\
Ladder resistances, $R_{3 a}, R_{3 b}$ & $6.952 \cdot 10^{-4}, 0.0024 \Omega$ & $6.67 \cdot 10-4,0.0024 \Omega$ \\
Ladder resistances, $R_{4 a}, R_{4 b}$ & $9.41 \cdot 10^{-5}, 2.67 \cdot 10^{-4} \Omega$ & $8.95 \cdot 10^{-5}, 2.63 \cdot 10^{-4} \Omega$ \\
Ladder inductances, $L_{1 a}, L_{1 b}$ & $3.4 \cdot 10^{-10}, 1.4 \cdot 10^{-11} \mathrm{~F} / \mathrm{m}$ & $3.3 \cdot 10^{-10}, 1.39 \cdot 10^{-11} \mathrm{~F} / \mathrm{m}$ \\
Ladder inductances, $L_{2 a}, L_{2 b}$ & $4.5 \cdot 10^{-9}, 2.5 \cdot 10^{-10} \mathrm{~F} / \mathrm{m}$ & $4.48 \cdot 10^{-9}, 2.48 \cdot 10^{-10} \mathrm{~F} / \mathrm{m}$ \\
Ladder inductances, $L_{3 a}, L_{3 b}$ & $6.05 \cdot 10^{-8}, 4.4 \cdot 10^{-9} \mathrm{~F} / \mathrm{m}$ & $6.04 \cdot 10^{-8}, 4.41 \cdot 10^{-9} \mathrm{~F} / \mathrm{m}$ \\
\hline \hline
\end{tabular}

\section{SIMULATION RESULTS AND DISCUSSION}

The effectiveness of the EMTR method is evaluated in two medium voltage (MV) power lines formed by coaxial cables with the characteristic shown in Table 1. The first line is homogeneous, $500 \mathrm{~m}$ long and realized with cable 1 , using XLPE insulation, and the second line, $500 \mathrm{~m}$ long, is formed by two inhomogeneous cables, i.e. cable $1,200 \mathrm{~m}$ long, and cable $2,300 \mathrm{~m}$ long, with the same characteristic of cable 1 but with EPR insulation and the geometric characteristic shown in Table 1. Then, two cable sections have a different shunt capacitance per unit length, hence a different characteristic impedance and propagation speed, as shown in Table 2.

For the two transmission lines, a lossy TLM model is developed, as described in Section 3, to perform the simulation of the PD signal propagation in the direct time domain.

PD signals are impulsive signals with frequency components up to several $\mathrm{MHz}[3]$. Time domain PD location methods are based on the measurement of single PD pulses along the line, using wide bandwidth detectors up to several tens of $\mathrm{MHz}$ [2][28], such as the HFCT sensors with bandwidth from a few hundreds of $\mathrm{kHz}$ to $30 \mathrm{MHz}$ ). Moreover, it has been experimentally verified that the propagation function, in extruded cables, shows a strong non-linear behaviour caused by the skin effect in a frequency region of DC to a few $\mathrm{MHz}$ [18]. For these reasons, a maximum frequency of interest, $f_{m}$, equal to $50 \mathrm{MHz}$ has been chosen to evaluate the parameters $\alpha_{R}$ and $\alpha_{L}$ of relations (19) and the ladder characteristics of the TLM skin effect model, that are shown in table 2. Figures 8 shows the frequency behavior of the resistance and inductance of the distributed complex impedance of the cable 1, reproduced by the developed TLM model, compared with the theorical values obtained by relations (16). As the figure shows, the TLM model of the distributed impedance follows the behavior of the theorical one.

Figures 9 (at the top) shows the distortion of a PD signal (represented with the double exponential pulse) during its
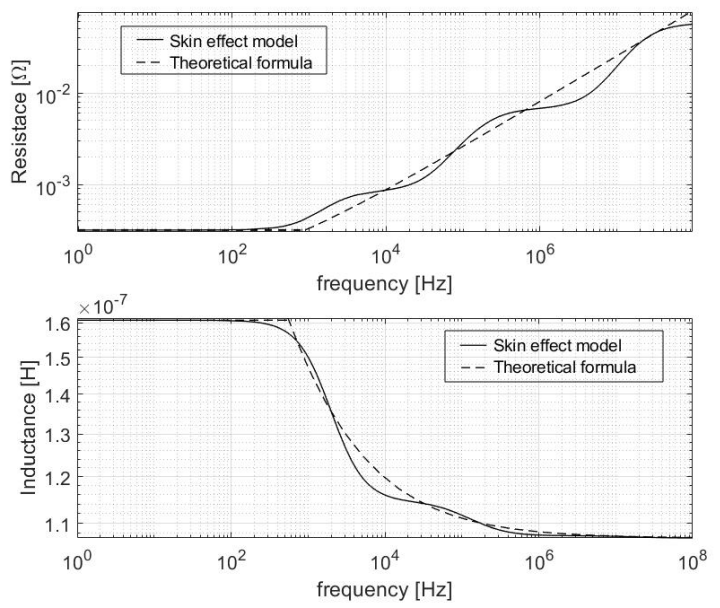

Fig. 8. Comparison of the cable resistance (top) and inductance (bottom) obtained with the skin effect model and with the theoretical formula.

propagation along the modelled coaxial line. The figure shows the pulse at the beginning of the line (the injection point) and the pulse recorded at $150 \mathrm{~m}$ and at $260 \mathrm{~m}$ along the line. The distortion of the signal shape due to the nonlinear behavior of the attenuation and phase velocity of the line, caused by the skin effect, is clearly shown. Figures 9 (at the bottom) shows the FFT of the recorded signals, where it is possible to see that the higher frequency components of the signal are strongly attenuated reducing its bandwidth.

The EMTR method was then tested using the TLM model with skin effect of the homogeneous and inhomogeneous line to perform the DT simulation. During the DT simulation, a PD event has been considered occurring at a point, $x_{P D}$, of the line. At the OP, located at the left end of the line, as shown in figure 6 , the PD signals are recorded for a period, $T$, useful to record the direct PD signal coming from the PD source and some reflections from the right end of the line. The amount of the reflected signals is defined by the reflection coefficient of the line that, in this case, is close to 1 because the load impedances at the cable ends are much higher than the characteristic impedances of the lines. Then, the recorded signal is time reversed and injected in the lossless 1D TLM model of the line and the TR simulations are performed to locate the PD source as described in Section 4. To locate the PD source, a first scan of the line is performed choosing GPDLs 4m apart from each
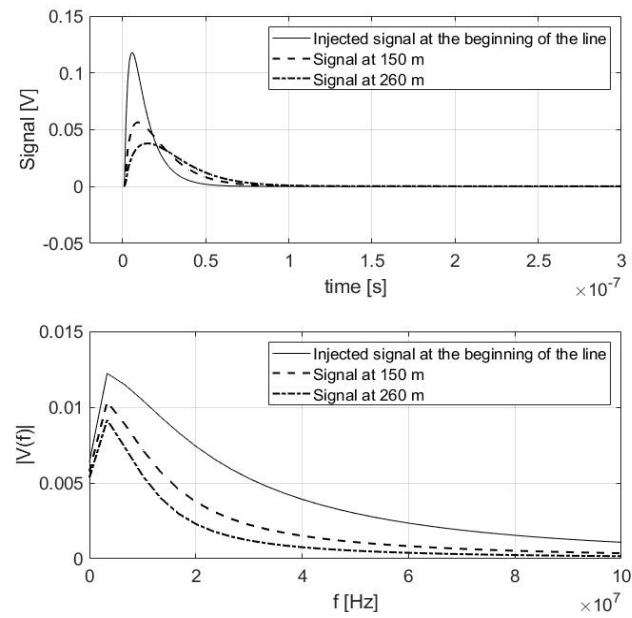

Fig. 9. Signal in ejected at the beginning of the line and measured at $150 \mathrm{~m}$ and $260 \mathrm{~m}$ along the lines (top) and their FFTs (bottom). 
other along the line. Then, a refined search is performed reducing the distance between the GPDLs to $1 \mathrm{~m}$ only in the section of the line where the first scanning has revealed the maximum concentration of the energy in the GPDL shunt impedances associated to the time reversed signals propagation. Figures 10 and 11 show the results of the location method used in the homogeneous line, when the PD sources are, respectively, at $124 \mathrm{~m}$ and $380 \mathrm{~m}$ from the left end of the line.

The figures show the normalized energy evaluated at the GPDLs using relation (30). As the figures show, the method is able to locate the PD source with an error $<1 \%$.

Figures 12-14 show the effectiveness of the method to localize PD sources in a line with inhomogeneous cable sections. Figure 12 shows the results when the source in the first section of the line, with the PD located at $175 \mathrm{~m}$ from the line left end. Figures 13 and 14, the results when the PDs are in the second section of the line, respectively at $420 \mathrm{~m}$ and $245 \mathrm{~m}$ from the left. The errors in the localization are $\leq 1 \%$ also in this case.

In figure 15 , the error of the method to localize PD source versus the position of the PD source along the line is reported. As the figure shows, the error is always lower than $1 \%$ in the middle of the line and always $\leq 1.5 \%$ in proximity of the line terminations. This is because, when a PD occurs close to the observation point or to a cable end, the direct and reflected signals are very close and mix, somewhat increasing the error in the evaluation of the point with the maximum energy.

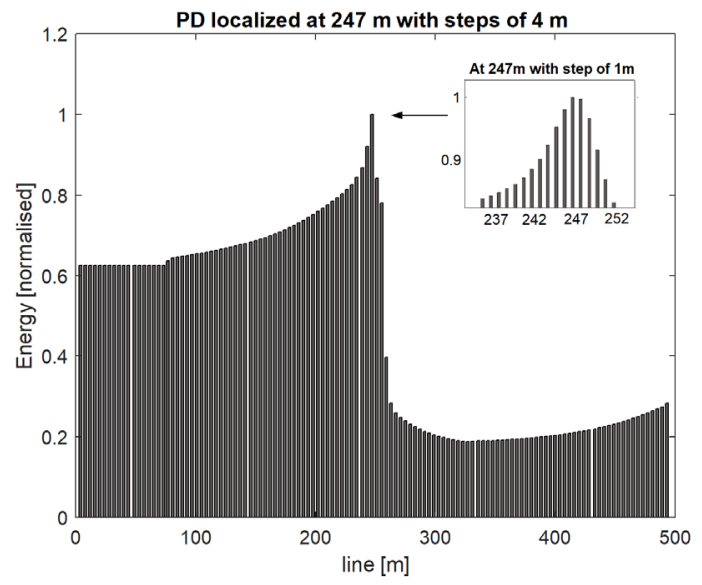

Fig. 10. Normalized energy when the PD source is at $250 \mathrm{~m}$ in the homogeneous line.

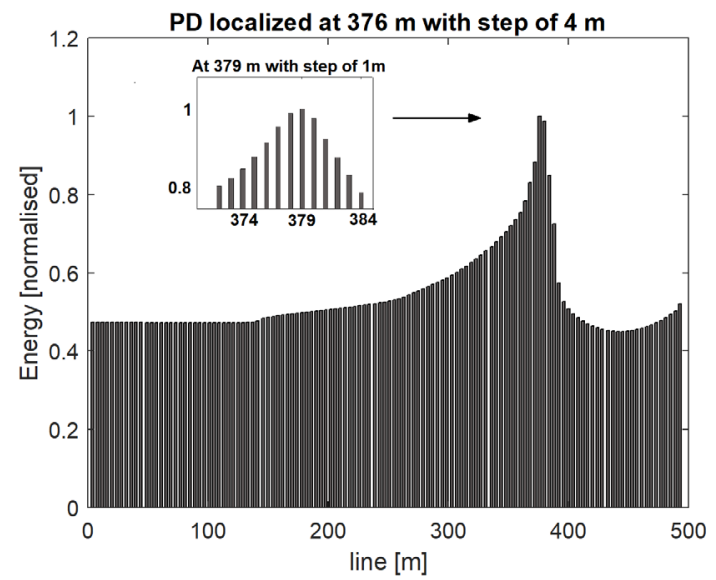

Fig. 11. Normalized energy when the PD source is at $380 \mathrm{~m}$ in the homogeneous line.

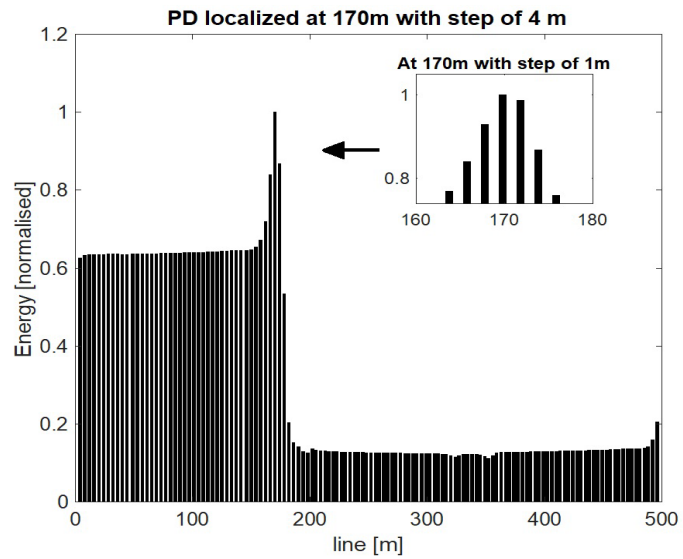

Fig. 12. Normalized energy when the PD source is at $175 \mathrm{~m}$ in the inhomogeneous line.

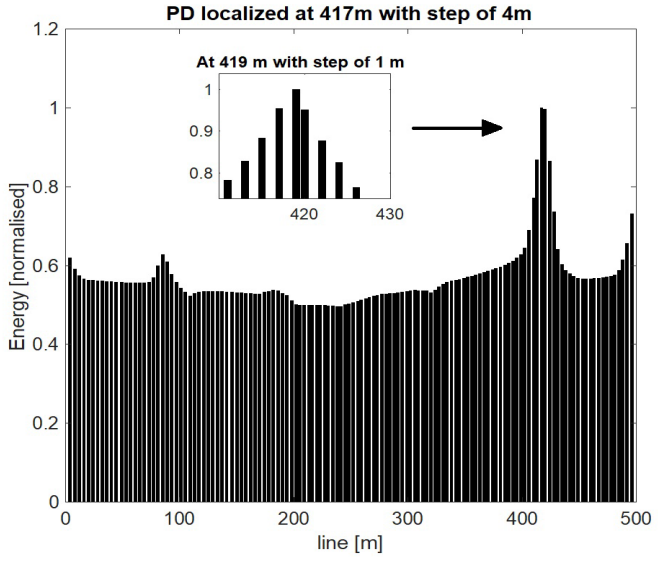

Fig. 13. Normalized energy when the PD source is at $420 \mathrm{~m}$ in the inhomogeneous line.

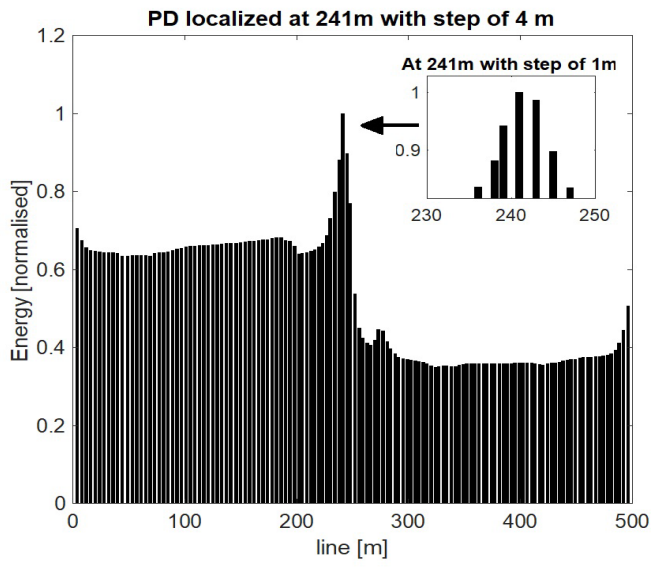

Fig. 14. Normalized energy when the PD source is at $245 \mathrm{~m}$ in the inhomogeneous line.

\section{CONCLUSIONS}

This paper presents the numerical validation of a new method to locate PD on power networks, based on the EMTR theory and on the use of a 1D TLM lossless model of the line to perform the simulations in the TR domain. The effectiveness of the method and its accuracy are analyzed. To this aim, a lossy TLM model of the line has been used to perform the simulation of the PD signal propagation in the direct time (DT) domain. The lossy model is able to reproduce the distortion of PD signals during the propagation. The recorded signals from the DT simulation, affected by distortion like the measured ones in 


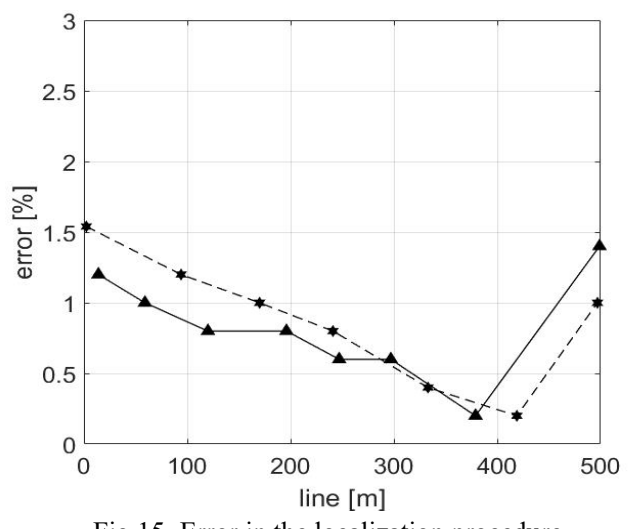

Fig.15. Error in the localization procedure.

a real system, are used as input signals in the TR simulations used to locate the PD source. The lossy TLM model of the line is presented and theoretically validated. The analysis of the method's effectiveness shows that the method is able to locate PDs in power lines, also with inhomogeneous cable sections, with a relative error, with respect to the length of the line, always lower than $1.5 \%$. The numerical tools proposed in this paper allows us to investigate also the effects of the noise and joints present in real networks, the sensors used to measure the PD signal, and of the quantization and sampling errors on the DT PD signals. The mentioned analysis will be developed both numerically and experimentally in future works. Moreover, the effectiveness of the proposed method to localize more than one PD sources in the same line section is also under analysis.

\section{REFERENCES}

[1] S. Refaat, M. Sham - "A Review of Partial Discharge Detection, Diagnosis Techniques in High Voltage Power Cables" - IEEE Int. Conf. on Comp. Pow. Elect. and Power Eng., 2018.

[2] EN 60270 - High-voltage test techniques - Partial discharge measurements (IEC 60270:2000) - 2001.

[3] F. Auzanneau, "Wire Troubleshooting and diagnosis: Review and perspectives" - Progress in Electromagnetics Research B, Vol. 49, 2013.

[4] W. He, Q. Wang, C. Huang, H. Li, D. Liang, "A cost-effective technique for PD testing of MV cables under combined AC and damped AC voltage", IEEE Trans. Power Del., vol. 33, no. 4, Aug. 2018.

[5] C. C. Yii, M. N. K. H. Rohani, M. Isa, and S. I. S. Hassan, "Multi-end PD location algorithm using segmented correlation and trimmed mean data filtering techniques for MV underground cable," IEEE Transactions on Dielectrics and Electrical Insulation, vol. 24, no. 1, Feb. 2017.

[6] F. P. Mohamed, W. H. Siew, J. J. Soraghan, S. M. Strachan, "Partial Discharge Location in Power Cables using a Double Ended Method Based on Time Triggering with GPS", IEEE Transactions on Dielectrics and Electrical Insulation, Vol. 20, No. 6; Dec 2013.

[7] Z. Du, P. K. Willett, M. S. Mashikian - "Performance Limits of PD Location Based on Time-domain Reflectometry"- IEEE Trans. on Dielect. and Elect. Insulation, Vol. 4, No. 2, April 1997.

[8] G. Robles, M. Shafiq, J. M. Martínez-Tarifa, "Multiple Partial Discharge Source Localization in Power Cables Through Power Spectral Separation and Time-Domain Reflectometry", IEEE Trans, on Instrumentation and Measurement, Vol. 68, No. 12, Dec. 2019.

[9] G. Zhu; K. Zhou; S. Zhao; Y. Li; L. Lu, "A Novel Oscillation Wave Test System for Partial Discharge Detection in XLPE Cable Lines", IEEE Trans. Power Del, Vol. 35, no. 4, pp. 1678-1684 Aug. 2020.

[10] M. Mahdipour, A. Akbari, P. Werle, H. Borsi, "Partial Discharge Localization on Power Cables Using On-Line Transfer Function", IEEE Trans. Power Del., Vol. 34, No. 4, pp. 1490 - 1498, Aug. 2019.

[11] G. Fulli, F. Profumo, and E. Bompard, Electricity Security in the EU: Features and Prospects, Joint Res. Centre, Brussels, Belgium, Aug. 2018.

[12] J. Zhon, X.Bi, Q. Shu, , M. Chen, D. Zhou, D. Zhang, "Partial Discharge Signal Denoising Based on Singular Value Decomposition and Empirical Wavelet Transform" IEEE Trans, On Instrumentation and Measurement, Vol. 69, No. 11, Nov. 2020.
[13] S. Zhou, J. Tang, C. Pan, Y. Luo, K. Yan, "Partial Discharge Signal Denoising Based on Wavelet Pair and Block Thresholding", IEEE Access, June 30, 2020.

[14] F. Rachidi, M. Rubinstein, M. Paolone, "Electromagnetic Time Reversal - Application to Electromagnetic Compatibility and Power System" John Wiley \& Sons Ltd, 2017; pp. 95-97.

[15] R. Razzaghi, G. Lugrin, H. Manesh, C. Romero, M. Paolone, F. Rachidi, An Efficient Method Based on the Electromagnetic Time Reversal to Locate Faults in Power Networks - IEEE Trans. on Pow. Del., Vol. 28, No. 3, July 2013.

[16] H. Karami, M. Azadifar, A. Mostajabi, M. Rubinstein, F. Rachidi, "Numerical and Experimental Validation of Electromagnetic Time Reversal for Geolocation of Lightning Strikes" IEEE Tran. on EMC, Vol. 62, No. 5, Oct. 2020.

[17] S.Y. He, Y.Z. Xie, Z. Wang, F. Rachidi, B.Y. Liu, Q Li, X. Kong "Norm Criteria in the Electromagnetic Time Reversal Technique for Fault Location in Transmission Lines “, IEEE Trans. on EMC, Vol. 60, No. 5, pp. 1240 - 1248, Oct. 2018.

[18] A. Ragusa, H. Sasse, A. Duffy, F. Rachidi, M. Rubinstein "Electromagnetic Time Reversal Method to Locate Partial Discharges in Power Networks using 1D TLM modelling" - IEEE Letters on Electromagnetic Compatibility Practice and Applications, Nov. 2020.

[19] Qin Shaozhen and S. Birlasekaran - The Study of PD Propagation Phenomenon in Power Network - IEEE Tran. on Pow. Del., Vol. 21, No. 3, July 2006.

[20] P. C. Magnusson, G. C. Alexander, V. K. Tripathi, A. Weisshaar, Transmission lines and wave propagation, $4^{\text {th }}$ Ed., CRC Press LLC, 2001.

[21] M. Tozzi, A. Cavallini, G. C. Montanari, G. L. Giuliattini Burbui "PD Detection in Extruded Power Cables: An Approximate Propagation Model", IEEE Trans, on Diel. and Elec. Ins. Vol. 15, No. 3; June 2008.

[22] S. Ramo, J. R. Whinnery, T. Van Duzer, Fields and waves in communication electronics-Third Edition, John Wiley \& Sons Inc, 1994.

[23] C. Christopoulos, The transmission-line modeling method - TLM, Institute of Electrical and Electronics Engineers, 1995.

[24] S. Kim, D. P. Neikirk, "Compact Equivalent Model for the Skin Effect", Microwave Symposium Digest, 1996, IEEE MTT-S Inter. 1, Vol. 3.

[25] Y. X. Teo, Modelling of interconnects including coaxial cables and multiconductor lines. PhD thesis, University of Nottingham, 2013.

[26] A. Ragusa, H. Sasse, A. Duffy, Towards Modeling Partial Discharge Phenomena and Propagation in Power Networks Using the Transmission Line Matrix Method, Energies 2021, 14(3), 689; 29 Jan. 2021.

[27] L. Niemeyer, "A generalized approach to partial discharge modeling," IEEE Trans. on Diel. and El. Ins., vol. 2, no. 4, pp. 510-528, Aug. 1995

[28] IEEE Guide for Partial Discharge Testing of Shielded Power Cable Systems in a Field Environment. IEEE Std 400.3-2006, 5 Feb. 2007.

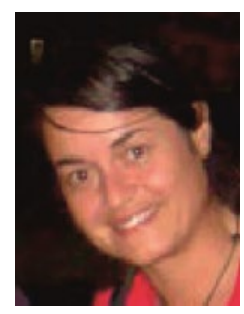

Antonella Ragusa, (M'08) received the Master and Ph.D. degrees in Electrical Engineering from the University of Palermo, Italy, in 2001 and 2006, respectively. In 2007, she worked at FIAT. She has been a permanent researcher at the Institute of Marine Engineering (INM) of National Research Council (CNR) of Italy, Palermo since 2008. Currently, she is a Marie Curie Researcher Fellow (MSCA-IF) at De Montfort University of Leicester, UK. Her research interests include electromagnetic compatibility, computational electromagnetics and smart grids

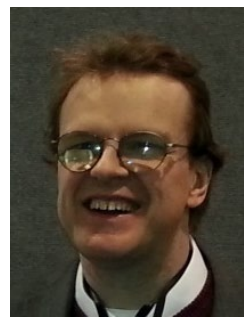

Hugh G. Sasse, received the B.Sc. (Hons) degree in electronic engineering from the University of York, York, U.K., in 1985, and has received his $\mathrm{PhD}$. degree in 2010 from De Montfort University, Leicester, U.K. His research is on optimization of physical layer components for communications systems at De Montfort University. 
Alistair Duffy, (SM'04, F'14), is Professor of Electromagnetics and

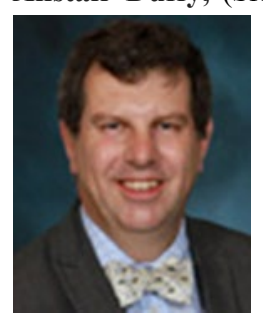

Director of the Institute of Engineering Sciences at

De Montfort University (DMU), Leicester, UK.

He received his BEng (Hons) and MEng degrees

in 1988 and 1989, respectively, from University

College, Cardiff, University of Wales. He read for

his $\mathrm{PhD}$ with professors Christopoulos and Benson

at Nottingham University, graduating in 1993. He

also holds an MBA from the Open University, UK,

graduating in 2004. He was awarded his DSc from Cardiff University in 2019 for his body of research on the validation of computational electromagnetics. He is a Fellow of the IEEE and President of the IEEE EMC Society. He has published approximately 300 papers, mostly on his research interests of validation of computational electromagnetics; physical layer components, particularly communications cabling, and electromagnetic compatibility testing. 